\title{
CD147 and downstream ADAMTSs promote the tumorigenicity of Kaposi's sarcoma-associated herpesvirus infected endothelial cells
}

\author{
Lu Dai ${ }^{1,3}$, Jimena Trillo-Tinoco4${ }^{4}$, Yihan Chen ${ }^{1}$, Karlie Bonstaff ${ }^{3}$, Luis Del Valle ${ }^{4}$, Chris \\ Parsons $^{3}$, Augusto C. Ochoa ${ }^{5}$, Jovanny Zabaleta ${ }^{5}$, Bryan P. Toole ${ }^{6}$, Zhiqiang Qin ${ }^{1,2}$ \\ ${ }^{1}$ Research Center for Translational Medicine and Key Laboratory of Arrhythmias, East Hospital, Tongji University School of \\ Medicine, Shanghai 200120, China \\ ${ }^{2}$ Departments of Microbiology/Immunology/Parasitology, Louisiana State University Health Sciences Center, Louisiana \\ Cancer Research Center, New Orleans, LA 70112, USA \\ ${ }^{3}$ Department of Medicine, Louisiana State University Health Sciences Center, Louisiana Cancer Research Center, New Orleans, \\ LA 70112, USA \\ ${ }^{4}$ Department of Pathology, Louisiana State University Health Sciences Center, Louisiana Cancer Research Center, New \\ Orleans, LA 70112, USA \\ ${ }^{5}$ Department of Pediatrics, Louisiana State University Health Sciences Center, Louisiana Cancer Research Center, New \\ Orleans, LA 70112, USA \\ ${ }^{6}$ Department of Regenerative Medicine and Cell Biology, Medical University of South Carolina and Hollings Cancer Center, \\ Charleston, SC 29425, USA
}

Correspondence to: Zhiqiang Qin, e-mail: zqin@Isuhsc.edu

Keywords: KSHV, Kaposi's sarcoma, CD147, microarray

Received: August 26, 2015

Accepted: November 25, 2015

Published: December 12, 2015

\section{ABSTRACT}

Kaposi's sarcoma-associated herpesvirus (KSHV) is the etiologic agent of several human cancers, including Kaposi's sarcoma (KS), which preferentially arise in immunocompromised patients and lack effective therapeutic options. We have previously shown that KSHV or viral protein LANA up-regulates the glycoprotein CD147, thereby inducing primary endothelial cell invasiveness. In the current study, we identify the global network controlled by CD147 in KSHV-infected endothelial cells using Illumina microarray analysis. Among downstream genes, two specific metalloproteases, ADAMTS1 and 9, are strongly expressed in AIDS-KS tissues and contribute to KSHV-infected endothelial cell invasiveness through up-regulation of IL-6 and VEGF. By using a KS-like nude mouse model, we found that targeting CD147 and downstream ADAMTSs significantly suppressed KSHV-induced tumorigenesis in vivo. Taken together, targeting CD147 and associated proteins may represent a promising therapeutic strategy against these KSHV-related malignancies.

\section{INTRODUCTION}

Kaposi sarcoma-associated herpesvirus (KSHV) represents one of major causative agent of cancers arising in immunocompromised patients, including Kaposi's Sarcoma (KS) [1]. Furthermore, despite the reduced incidence of KS in the era of combined Antiretroviral Therapy (cART) for Human Immunodeficiency Virus (HIV) infection, KS still remains the most common Acquired Immunodeficiency Syndrome (AIDS)associated tumor and a leading cause of morbidity and mortality in this setting $[2,3]$. In addition, a longitudinal study conducted among solid organ transplant recipients in United States reported a high prevalence (15\%) of
KSHV seropositivity in this population [4]. Transplant recipients who develop primary KSHV infection after transplantation have a relatively high probability of developing KSHV-related malignancies, especially KS [5], which is likely associated with the intensity of immunosuppressive treatment post-transplantation [6]. Therefore, KSHV-induced malignancies, in particular KS, still represent a serious threat to immunosuppressed patients due to the lack of effective therapies. In fact, KSHV has now become a model pathogen for virusinduced cancer research. However, many key questions regarding its mechanisms of oncogenesis still remain unanswered, thus hindering identification of rational targets and development of novel therapeutic strategies. 
The multifunctional transmembrane protein, CD147, also known as Emmprin or Basigin, induces the expression and secretion of multiple matrix metalloproteinases (MMPs), thereby promoting tumor cell invasion and other malignant behaviors [7-9]. We recently reported that enhancement of invasiveness for primary endothelial cells (the major cellular components of $\mathrm{KS}$ ), following de novo KSHV infection, resulted from up-regulation of CD147 by the KSHV-encoded latency-associated nuclear antigen (LANA) protein [10]. Further study indicated that PI3K/Akt and MAPK activation of vascular endothelial growth factor (VEGF) was required for CD147-mediated endothelial cell invasion [11]. In addition, CD147 and related proteins are also involved in multidrugresistance of primary effusion lymphoma (PEL), another KSHV-caused malignancy [12]. These data demonstrate the important role of CD147 in KSHV-associated malignancies. However, the global gene profile controlled by CD147 within primary endothelial cells, in particular KSHV-infected cells, remains unknown. It will also be interested to understand the cellular functions of CD147downstream proteins in vitro and in vivo, as well as their clinical relevance within AIDS-KS tumor tissues. In the current study, we used Illumina microarray to identify the global network controlled by CD147 within either CD147overexpressed or KSHV-infected endothelial cells. We also tested the contribution of two CD147-controlled proteins, ADAMTS (A Disintegrin and Metalloprotease with ThromboSpondin motifs) 1 and 9 to KSHV pathogenesis and their clinical correlations in AIDS-KS tissues. Finally, using a KS-like nude mouse model with KSHV long-terminfected, telomerase-immortalized human umbilical vein endothelial (TIVE-LT) cells [13], we assessed the role of CD147 and downstream ADAMTSs in KSHV-related tumorigenesis in vivo.

\section{RESULTS}

\section{Microarray analysis of the CD147 regulatory network in CD147-overexpressed and KSHV- infected endothelial cells}

We first used the HumanHT-12 v4 Expression BeadChip (Illumina), which contains more than 47,000 probes derived from the NCBI RefSeq Release 38 and other sources, to study the gene profile altered within CD147-overexpressed HUVEC cells by using a recombinant adenoviral vector AdV-CD147 [11] or within KSHV-infected HUVEC cells. We found that 184 genes were significantly up-regulated and 148 were down-regulated ( $\geq 2$ fold and $p<0.05$ ) within CD147overexpressed endothelial cells; in KSHV-infected cells, 963 genes were up-regulated and 1042 down-regulated. Intersection analysis indicated that 71 "common" genes were significantly up-regulated and 75 were downregulated in both sets (Figure 1A); the top 10 up-regulated and down-regulated candidate genes were listed in Table 1, respectively. We next selected 5 "common" genes in both sets from Table 1 for validation of their transcriptional change by using qRT-PCR. Our results indicated that all of the 10 selected genes were significantly altered in a manner comparable to those found in the microarray data, demonstrating the credibility of our microarray analysis. Specifically, ADAMTS1, ADAMTS9, HMOX1, TRIB1 and $I L-6$ were significantly up-regulated, while $Z n T 3$, GDF3, FBLN5, COL1A2, SDPR were significantly downregulated within either CD147-overexpressed or KSHVinfected endothelial cells (Figure 1B-1C).

Interestingly, some of the top altered candidate genes listed have been reported to be closely associated with KSHV pathogenesis. For example, KSHV infection induces heme oxygenase-1 (HMOX-1 or HO-1), an inducible enzyme responsible for the rate-limiting step in heme catabolism, in infected endothelial cells and/or AIDS-KS tissues [14]. Increased HMOX-1 enzymatic activity in vitro has been shown to enhance proliferation of KSHV-infected endothelial cells in the presence of free heme. Fibulin-5 (FBLN5), one of the most down-regulated genes, is greatly decreased in KSHVinfected endothelial cells and/or AIDS-KS tissues, while addition of recombinant Fibulin-5 suppresses VEGF production by KSHV-infected endothelial cells [15]. In contrast, some other candidates have never been reported in KSHV pathogenesis but are thought to be involved in progression of other cancers, such as ADAMTS1 and 9. The ADAMTS family of extracellular metalloproteases, including ADAMTS1 and 9, has been widely implicated in remodeling of the tumor microenvironment during cancer development, growth and progression [16-19]. In particular, elevated ADAMTS1 promotes pro-tumorigenic changes such as increased tumor cell proliferation, decreased apoptosis and altered vascularization [20]. Importantly, it facilitates significant peritumoral remodeling of the extracellular matrix (ECM) microenvironment to promote tumor progression and metastasis. For these reasons we chose ADAMTS1 and 9 for further investigations.

We also performed enrichment analysis of the "common" genes in both sets by using the Pathway map, Gene Ontology (GO) Processes and Process Networks modules from Metacore Software (Thompson Reuters) [21]. Our analysis showed that these genes belong to several major cellular function categories, including cellular immune response to inflammation, blood vessel development, regulation of epithelial-mesenchymal transition (EMT), cell adhesion and cell cycle/proliferation (Supplementary Figure S1A-S1C), some of which have been reported closely related to $\mathrm{KSHV}$ pathogenesis or tumorigenesis $[22,23]$. In addition, the top 2 scored pathway maps and protein networks for these "common" genes were shown in Supplementary Figure S2 and Figure S3, respectively. 


\section{Clinical relevance of CD147 and downstream ADAMTSs within AIDS-KS tissues}

Due to the important role of ADAMTS family members in cancer progression, we further examined their regulation by CD147 in endothelial cells and their expression in AIDS-KS tumors. We previously demonstrated up-regulation of CD147 expression in HUVEC by either a recombinant CD147 adenovirus (AdV-CD147) transduction or KSHV de novo infection $[10,11]$. Here we showed the elevated expression of ADAMTS1 and 9 after both treatments in cultured HUVEC cells (Figure 2A-2B). Furthermore, we measured their expression levels in KS tumor tissues directly isolated from cohort 3 AIDS patients without chemotherapy treatments. Our results showed the strong expression of CD147, as well as ADAMTS1 and 9 within KS tumor tissues from all the patients (CF0002, JG004 and XX007) (Figure 2C). In addition, we found that CD147, ADAMTS1 and 9 were all expressed mostly in "spindle cells", the typical LANA+ KS tumor cells [24] (Figure 2D). Taken together, these data strongly suggest the involvement of CD147 and downstream ADAMTS proteins in KSHV-related malignancies, specifically KS.
Targeting CD147 and downstream ADAMTSs represses pro-inflammatory, pro-angiogenic cytokine production and endothelial cell invasion

Pro-inflammatory and pro-angiogenic cytokines, in particular IL-6 and VEGF, are secreted by KSHV-infected cells. Their presence within KS lesions and the peripheral circulation of KS patients is thought to facilitate KSHVassociated cellular pathogenesis and angiogenesis [25-27]. Moreover, acquisition of a migratory or invasive phenotype represents one hallmark of KSHV-infected endothelial cells, with implications for both viral dissemination and angiogenesis within $\mathrm{KS}$ lesions [28]. Here our results indicated that targeting CD147, ADAMTS1 or 9 by RNAi significantly reduced IL-6 and VEGF production from KSHV-infected HUVEC, as well as the transcripts for their receptors (IL-6R, VEGFR1 and VEGFR2) (Figure 3A-3C and Supplementary Figure S4). By using the transwell assays, we found that silencing of CD147, ADAMTS1 or 9 effectively reduced KSHV-infected HUVEC invasion (Figure 3D). Moreover, silencing of CD147, ADAMTS1 or 9 also significantly reduced CD147-overexpressed HUVEC invasion (Figure 3E), demonstrating that these ADAMTSs are indeed required for CD147-mediated endothelial cell invasion.
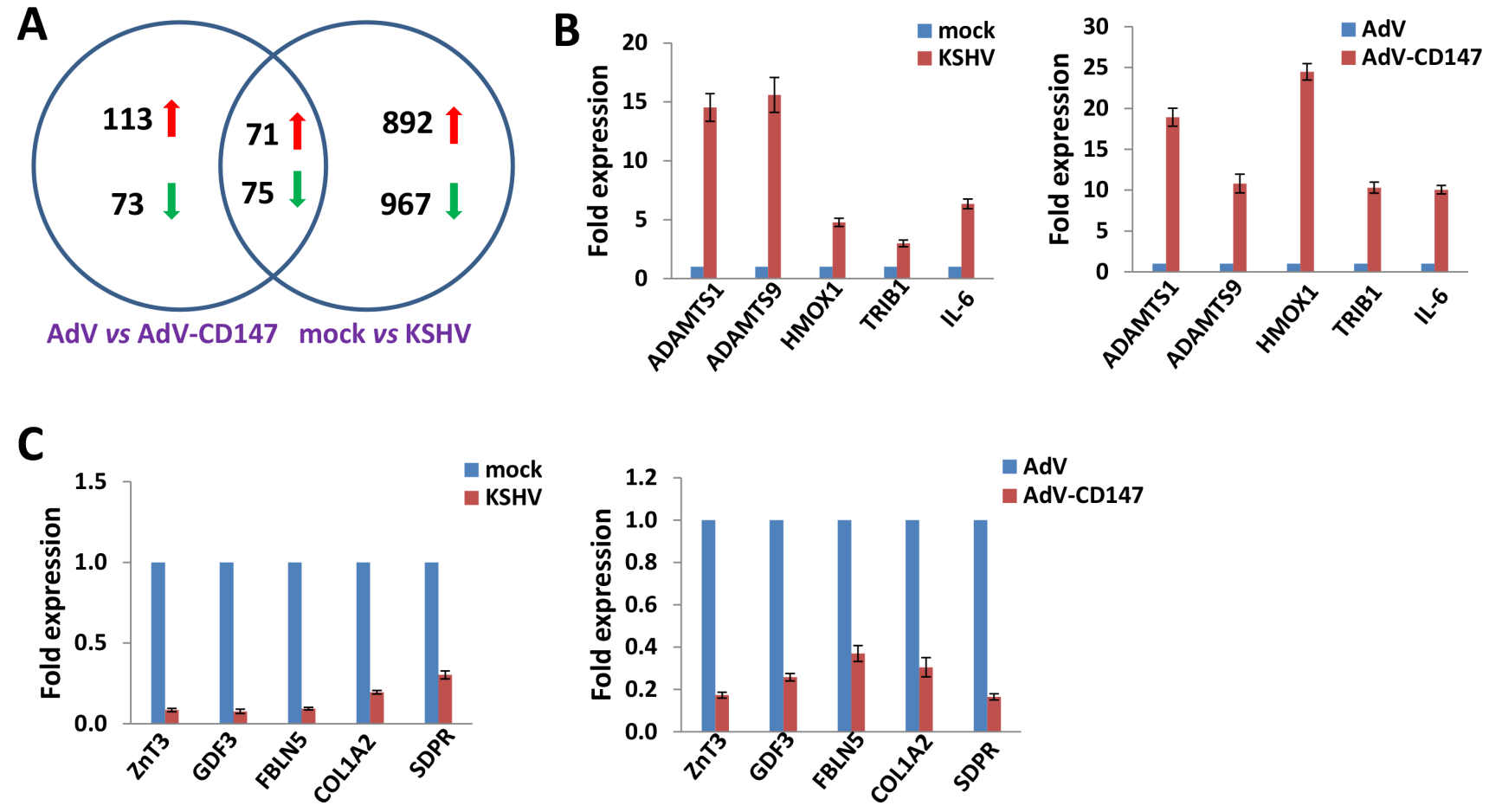

Figure 1: Intersection analysis and experimental validation of gene profile alterations in KSHV-infected and CD147overexpressed endothelial cells. (A) The HumanHT-12 v4 Expression BeadChip (Illumina) was used to detect alterations in gene profile in HUVEC cells infected by KSHV ( $v s$ mock cells), or cells transduced with AdV-CD147 ( $v s$ AdV-transduced cells). Intersection analysis of significantly altered genes (up/down $\geq 2$ fold and $p<0.05$ ) was performed using the Illumina GenomeStudio Software. (B-C) The transcriptional levels of 5 selected 'common' candidate genes that were up-regulated (B) or down-regulated (C) in each set of microarray data were validated by using qRT-PCR. Error bars represent the S.E.M. for 3 independent experiments. 
Table 1: The top 10 'common' candidate genes upregulated or downregulated within both CD147overexpressed and KSHV-infected HUVEC cells

\begin{tabular}{|c|c|c|c|}
\hline \multirow[b]{2}{*}{ Gene symbol } & \multirow[b]{2}{*}{ Description } & \multicolumn{2}{|c|}{ Fold change } \\
\hline & & $\begin{array}{c}\text { CD147- } \\
\text { overexpressed }\end{array}$ & KSHV-infected \\
\hline ADAMTS1 & $\begin{array}{l}\text { A disintegrin and metalloproteinase with } \\
\text { thrombospondin motifs } 1\end{array}$ & 16.72 & 19.26 \\
\hline HMOX1 & Heme oxygenase 1 & 25.8 & 2.31 \\
\hline RASD1 & Dexamethasone-induced Ras-related protein 1 & 19.74 & 2.21 \\
\hline ADAMTS9 & $\begin{array}{l}\text { A disintegrin and metalloproteinase with } \\
\text { thrombospondin motifs } 9\end{array}$ & 6.69 & 12.89 \\
\hline TMEM158 & Transmembrane protein 158 & 14.15 & 2.44 \\
\hline TRIB1 & Tribbles homolog 1 & 12.23 & 3.58 \\
\hline DUSP12 & Dual specificity protein phosphatase 12 & 12.8 & 2.81 \\
\hline IL6 & Interleukin-6 & 8.13 & 6.69 \\
\hline RGS2 & Regulator of G-protein signaling 2 & 3.73 & 10.64 \\
\hline CXCL2 & $\mathrm{C}-\mathrm{X}-\mathrm{C}$ motif chemokine 2 & 11.96 & 2.35 \\
\hline ZnT3 & Zinc transporter 3 & 0.13 & 0.13 \\
\hline GDF3 & Growth/differentiation factor 3 & 0.16 & 0.12 \\
\hline MYH10 & Myosin-10 & 0.03 & 0.26 \\
\hline LTBP2 & $\begin{array}{l}\text { Latent-transforming growth factor beta-binding } \\
\text { protein } 2\end{array}$ & 0.12 & 0.25 \\
\hline FBLN5 & Fibulin-5 & 0.24 & 0.15 \\
\hline COL1A2 & Collagen alpha-2(I) chain & 0.24 & 0.18 \\
\hline STAT1 & $\begin{array}{l}\text { Signal transducer and activator of transcription } \\
\text { 1-alpha/beta }\end{array}$ & 0.07 & 0.36 \\
\hline GPR126 & G-protein coupled receptor 126 & 0.2 & 0.23 \\
\hline GPR124 & G-protein coupled receptor 124 & 0.23 & 0.28 \\
\hline SDPR & Serum deprivation-response protein & 0.08 & 0.47 \\
\hline
\end{tabular}

\section{Establishment of a KS-like nude mouse model using TIVE-LT cells}

KSHV-infected primary endothelial cells usually cannot form tumors even in immunodeficiency mice [24]. Recently, a stable KSHV latency cell-line, named TIVE-LT cells has been established, which can induce KS-like tumor formation in nude mice [13]. Here we found robust LANA expression in the nuclei of TIVE-LT cells (a molecular marker for KSHV latency) [29], while none was observed in the uninfected parental TIVE cells (Figure 4A). Interestingly, TIVE-LT cells displayed much higher levels of CD147, ADAMTS1 and 9 expression than TIVE cells (Figure 4B). Accordingly, TIVE-LT cells possessed much stronger capacities for cell invasion and anchorage-independent growth than TIVE cells, the latter forming almost no colonies in the soft agar assay (Figure 4C-4D).

We next examined the ability of TIVE and TIVE-LT cells to form tumors after subcutaneous injection into nude mice. We found that all the mice injected with TIVE-LT cells developed visible tumors by 28 day (4/4), while none $(0 / 4)$ of the mice injected with TIVE cells did (Figure 4E). $\mathrm{H} \& \mathrm{E}$ staining of tumor tissues from TIVE-LT-injected mouse displayed KS-like features, including a mixture of elongated-spindle-cell and undifferentiated morphologies with prominent mitotic Figures. Immunohistochemistry (IHC) staining showed that most tumor cells expressed KS-specific viral/cellular marker molecules [24], such as LANA, CD31 and LYVE-1 (Figure 4F). 


\section{Targeting CD147 and downstream ADAMTS1} suppresses KSHV+ TIVE-LT cell tumorigenesis in vivo

Since TIVE-LT cells displaying high CD147 expression, we found that silencing of CD147 by RNAi reduced the expression of ADAMTS1 and 9 (Figure 5A), as well as their transcripts (data not shown). Silencing of CD147 by RNAi significantly blocked TIVE-LT cell invasion and anchorage-independent growth, when compared to negative control siRNA (n-siRNA) group (Figure 5B-5C). We also observed long-term "knockdown" of CD147 expression by RNAi for at least 2 weeks in vitro culture (Supplementary Figure S5). Next, we injected TIVE-LT cells transfected with n-siRNA or
CD147-siRNA subcutaneously into the right and left flanks of nude mice, respectively. These mice were observed and measured every $2 \sim 3$ day for the presence of palpable tumors for 21 days. Our results indicated that silencing of CD147 significantly repressed tumor growth in nude mice. Mice injected with CD147-siRNA formed smaller tumors when compared to n-siRNA groups at 21 days (Figure 5D-5E). Immunoblot results confirmed the higher levels of CD147, ADAMTS1 and 9 expression in tumor lysates from mice with n-siRNA than those from mice with CD147-siRNA, demonstrating the successful silencing of CD147 and downstream ADAMTSs in vivo (Figure 5F). Our additional data indicated that direct silencing of ADAMTS1 by siRNA also significantly repressed tumor growth in nude mice (Supplementary Figure S6). Taken
A

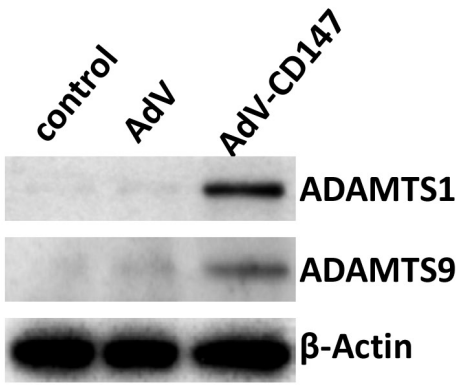

C
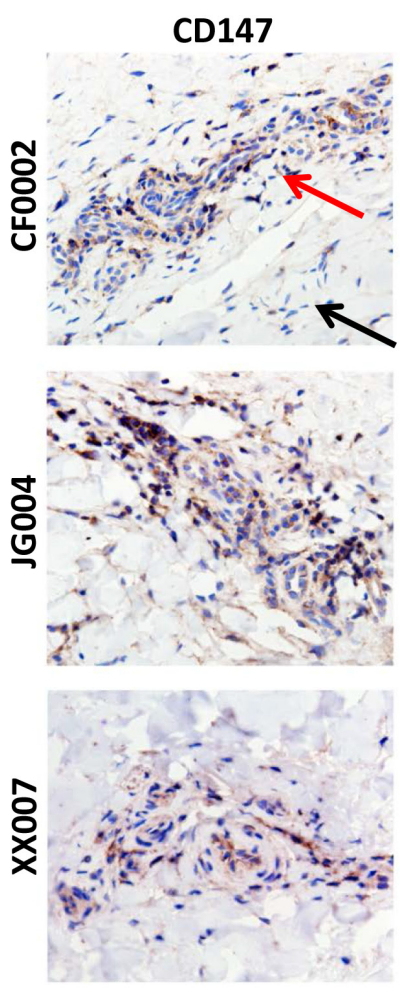

B

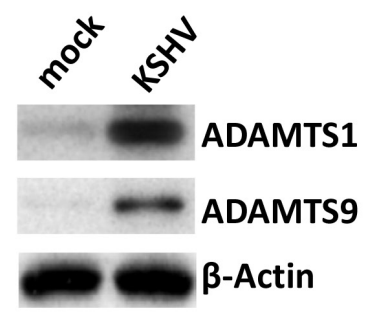

ADAMTS1
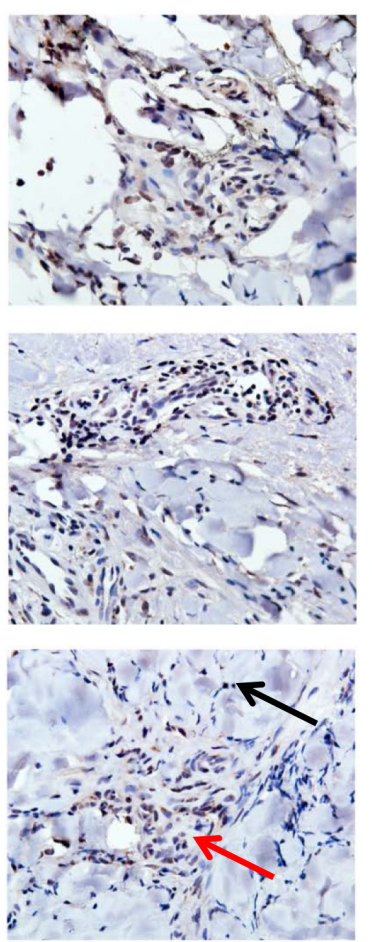
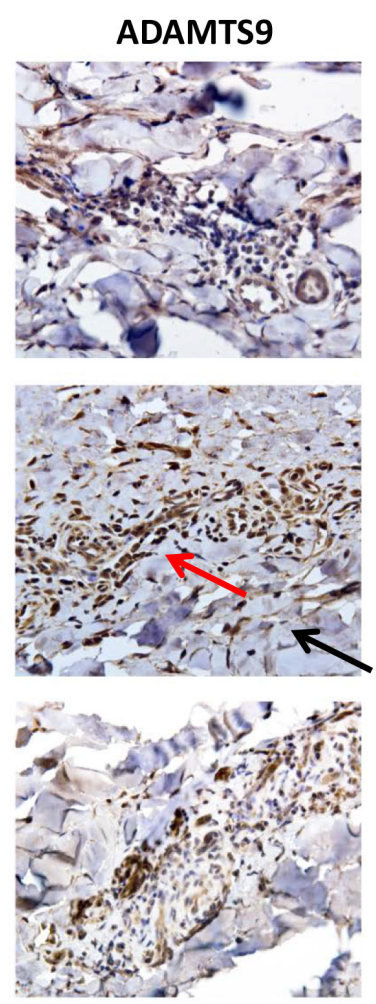

D
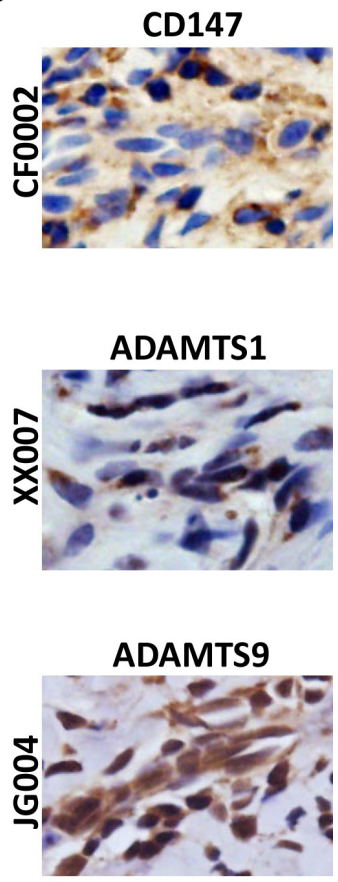

Figure 2: Up-regulation of ADAMTS1 and 9 expression in CD147-overexpressed or KSHV-infected endothelial cells and in AIDS-KS tissues. (A-B) HUVEC were transduced using a recombinant human CD147-encoding adenovirus (AdV-CD147), or control adenovirus (AdV) for $48 \mathrm{~h}$, or infected by purified KSHV (MOI 10) for $48 \mathrm{~h}$. Protein expression was measured by immunoblots. (C-D) The expression of CD147, ADAMTS1 and ADAMTS9 within KS tumor tissues from 3 cohort HIV+ patients was detected by immunohistochemistry (400x magnification, and tumor cells in selected regions were magnified at 600x in panel D). Red arrows indicate the KS tumor area and black arrows indicate the adjacent area from the same patient. 


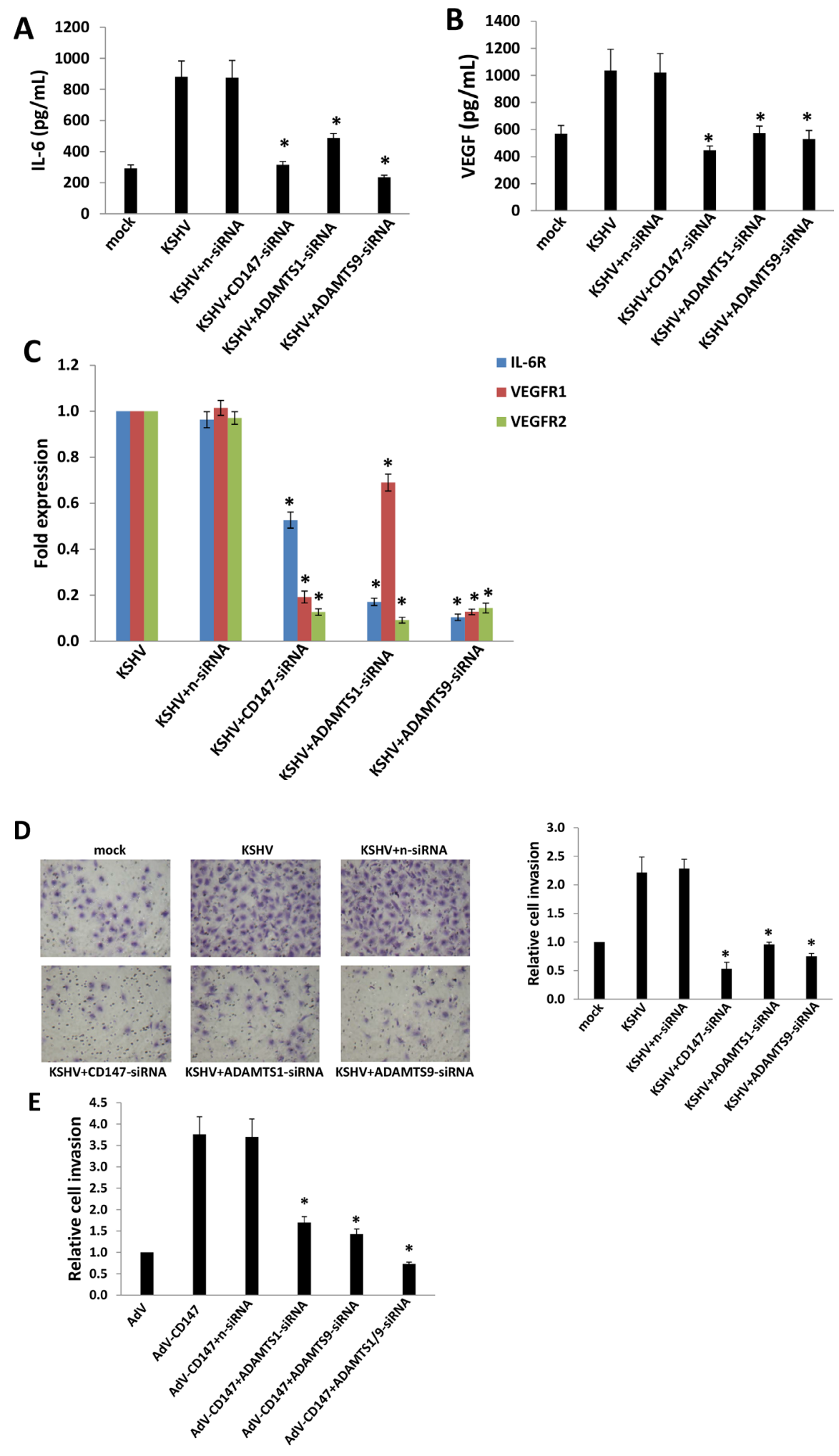

Figure 3: Targeting CD147 and downstream ADAMTSs represses expression of IL-6, VEGF, their respective receptors, and KSHV-infected endothelial cell invasion. (A-B) HUVEC were incubated with media (mock) or purified KSHV for $2 \mathrm{~h}$, with or without prior transfection for $48 \mathrm{~h}$ with negative control siRNA (n-siRNA), CD147-siRNA, ADAMTS1-siRNA or ADAMTS9-siRNA, respectively. Cells were subsequently incubated in fresh media for an additional $24 \mathrm{~h}$. The concentrations of IL-6 and VEGF in culture supernatants were determined using ELISAs. (C-D) HUVEC were treated as above, then the expression of cytokine receptor genes and cell invasiveness were measured by qRT-PCR and transwell assays, respectively. (E) HUVEC were transfected for $48 \mathrm{~h}$ with negative n-siRNA, ADAMTS1-siRNA or ADAMTS9-siRNA, respectively, then transduced with AdV-CD147 or control AdV for $48 \mathrm{~h}$. Cell invasion was assessed by transwell assays. Error bars represent the S.E.M. for 3 independent experiments. ${ }^{*}=p<0.05$. 
together, these data support the important role of CD147, ADAMTS1 and 9 as cellular co-factors for KSHV-related tumorigenicity and KS development.

\section{DISCUSSION}

Our previous studies have demonstrated that CD147 is an important contributor to KSHV-induced endothelial cell invasiveness, through a complex of underlying mechanisms $[10,11,30]$. However to our knowledge, there are no current data exploring the global gene profile controlled by CD147 in primary endothelial cells, in particular KSHV-infected cells, which are the major cellular components of KS tissues. In the current study, we identified at least $\sim 150$ genes potentially controlled by CD147 in KSHV-infected endothelial cells by using Illumina microarray. Interestingly, only a few of them have been reported associated with KSHV pathogenesis, e.g. HMOX-1 and FBLN5 $[14,15]$, while most of them remain functionally unknown with respect to KSHVrelated diseases. The enrichment analysis indicates that these genes belong to several major cellular function categories closely related to KSHV pathogenesis, such as EMT and blood vessel development [22, 23]. Although the role of EMT in KS development remains unclear, another similar pathophysiologic behavior, called as endothelial-to-mesenchymal transformation (EndMT), has been described during KSHV infection. Cheng et al. reported that KSHV-induced EndMT was initiated by the viral FLICE inhibitory protein (vFLIP) or vGPCR through Notch pathway activation, leading to cell invasiveness dependent upon membrane-type-1 matrix metalloproteinase (MT1-MMP) [31]. Gasperini et al. reported that canonical Notch signaling, including Slug

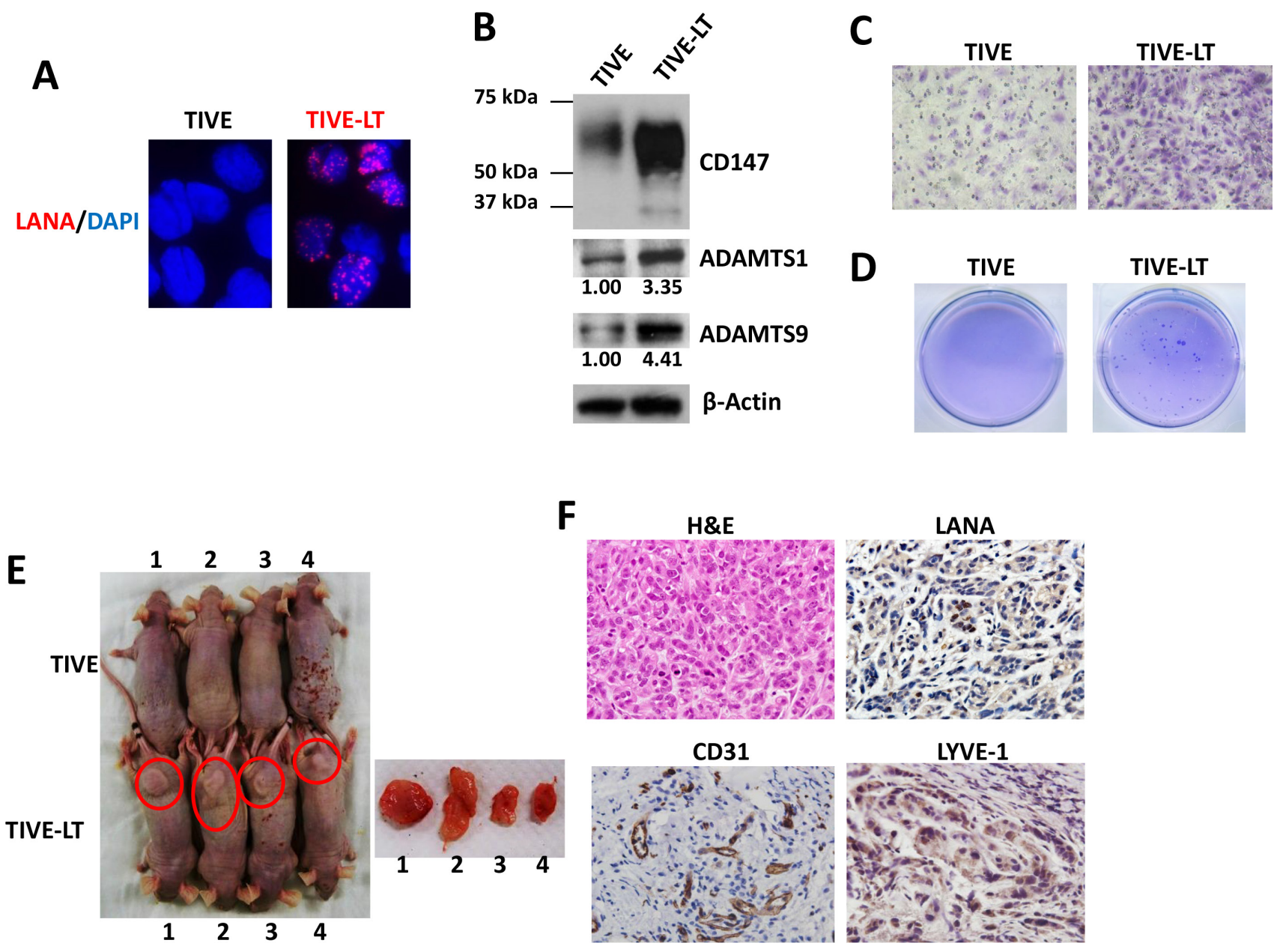

Figure 4: Establishment of a KS-like nude mouse model using TIVE-LT cells. (A) IFA was used to detect nuclear LANA expression in TIVE-LT cells, and TIVE cells as a negative control. (B-D) Protein expression (B), cell invasion (C) and anchorage-independent growth (D) were measured by immunoblots, transwell and soft agar assays, respectively. (E) TIVE or TIVE-LT cells (approximately $5 \times 10^{5}$ cells were mixed at a ratio of 1:1 with growth factor-depleted Matrigel) were injected subcutaneously into nude mice. Mice injected with TIVE-LT at $28 \mathrm{~d}$ developed tumors (4/4) as shown by red circle, while none (0/4) of the mice injected with TIVE cells did. (F) H \& E staining of representative tumor tissues isolated from TIVE-LT-injected mice displayed KS-like features, including a mixture of elongatedspindle-cell and undifferentiated morphologies with prominent mitotic figures. Immunohistochemistry (IHC) staining showed that most tumor cells expressed KS-specific viral/cellular marker molecules. 
and ZEB1, was required for KSHV-induced EndMT which increased invasiveness and survival in infected endothelial cells [32]. Interestingly, CD147 has been found involved in EMT and closely associated phenomena in cancer cells [33-35]. Additionally, the expression of functional CD147 and MMP-2 was significantly decreased in Notch1deficient breast cancer cells which displayed impaired migration and invasion [36].

Here we showed that two specific metalloproteases, ADAMTS1 and 9 were up-regulated by CD147 and contributed to KSHV-induced cell invasiveness. Previous reports showed that $\mathrm{CD} 147$ induced production of several MMPs [7-9] and urokinase-type plasminogen activator [37], but to our knowledge this is the first report showing the up-regulation of ADAMTSs by CD147. Moreover, CD147, ADAMTS1 and 9 were strongly expressed within AIDS-KS tumor tissues, demonstrating their potential clinical relevance as well as biomarker or therapeutic values. However, it remains unknown how CD147 can increase the expression and maturation of ADAMTS1 and 9, which may require a variety of additional factors. For example, ADAMTS1 is synthesized as a pro-zymogen and undergoes $\mathrm{N}$-linked glycosylation following protein translation [38]. The secretion of ADAMTS1 to the ECM requires the excision of its pro-domain from the $87 \mathrm{kD}$ mature protein by furin-related endopeptidases [38]. Interestingly, ADAMTS1 can be transiently induced by hypoxia in endothelial cells, which is mediated by hypoxia-inducible factor 1 (HIF-1) binding to its promoter region [39]. Moreover, CD147 can be induced in a similar manner in various carcinoma cells [40]. It has been reported that several KSHV-encoded proteins such as LANA, vIRF-3 or vGPCR can activate HIF-1 and contribute to angiogenesis, tumorigenesis or virus

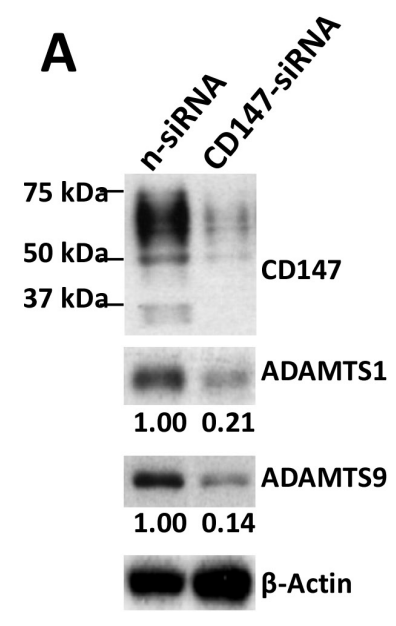

E

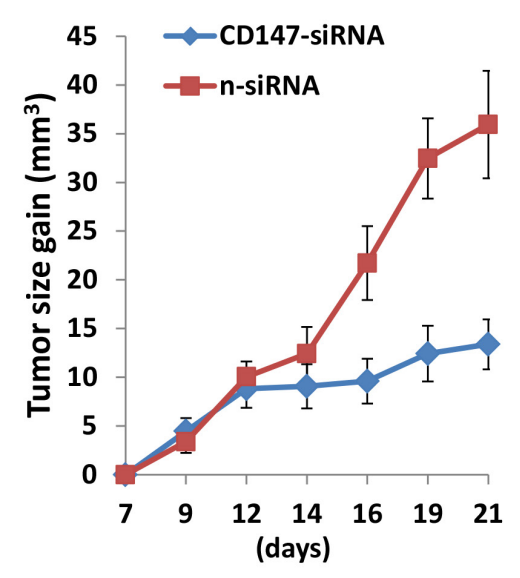

B
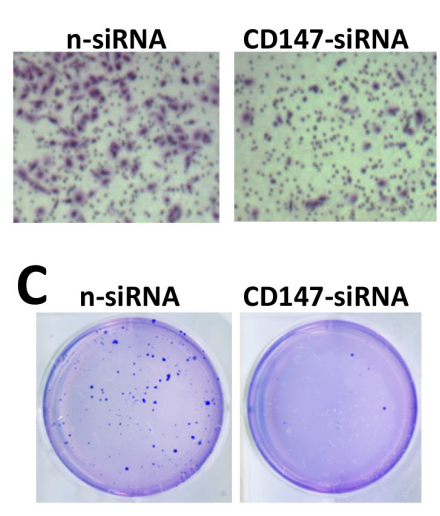

D

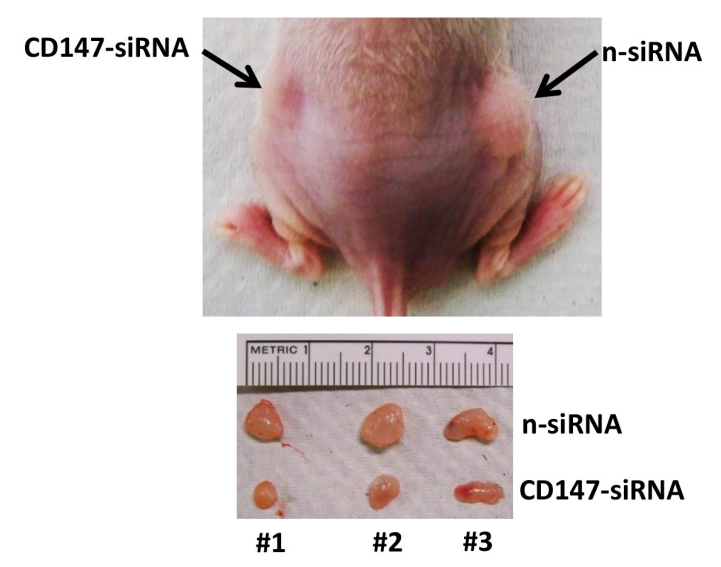

Figure 5: Targeting CD147 significantly suppresses TIVE-LT cell tumorigenesis in vivo. (A-C) TIVE-LT cells were transfected with negative control siRNA (n-siRNA) or CD147-siRNA for $48 \mathrm{~h}$, then protein expression (A), cell invasion (B) and anchorageindependent growth $(\mathrm{C})$ were measured by immunoblots, transwell and soft agar assays, respectively. (D-E) TIVE-LT cells transfected with n-siRNA or CD147-siRNA (approximately $5 \times 10^{5}$ cells were mixed at a ratio of 1:1 with growth factor-depleted Matrigel) were injected subcutaneously into the right and left flanks of nude mice ( 3 mice per group), respectively. The mice were observed and measured every $2 \sim 3 \mathrm{~d}$ for the presence of palpable tumors for $21 \mathrm{~d}$. (F) Protein expression was measured by immunoblots of tumor lysates from 2 TIVELT/n-siRNA or TIVE-LT/CD147-siRNA injected flanks of mice sacrificed at day 21, respectively. 
replication [41-44]. Therefore, we are interested to understand whether these viral proteins (latent or lytic) are able to up-regulate the expression of ADAMTS1 and 9 and the underlying mechanisms.

Our data showed that silencing of CD147 by RNAi significantly repressed tumor growth in a nude mouse model, and that these treated tumor tissues displayed less blood vessel formation and less tumor cells when compared to n-siRNA groups (data not shown). We assume this is partially due to impaired ECM dynamics in the tumor microenvironment through down-regulation of ADAMTS1 and 9. Our recent study indicated that silencing of CD147 by RNAi greatly reduced the production of hyaluronan, an important ECM component, through down-regulation of the hyaluronan synthase gene 1 (Hasl) within KSHV-infected HUVEC cells [30]. Interestingly, we found that silencing of CD147 also significantly reduced hyaluronan production from TIVE-LT cells, in this case through down-regulation of Has 2 and Has3 instead of Has 1 (Dai et al, unpublished data). Therefore, we will evaluate the strategies targeting ECM formation and/or components as potential therapies against KS development in future study.

\section{MATERIALS AND METHODS}

\section{Cell culture, reagents and infection protocol}

Body cavity-based lymphoma cells (BCBL-1, $\mathrm{KSHV}^{+} / \mathrm{EBV}^{-}$) were maintained in RPMI 1640 medium (Gibco) with supplements as described previously [45]. Telomerase-immortalized human umbilical vein endothelial (TIVE) and KSHV long-term-infected TIVE (TIVE-LT) cells were cultured as previously described [13]. Human umbilical vein endothelial cells (HUVEC) were grown in DMEM/F-12 50/50 medium (Cellgro) supplemented with 5\% FBS. All cells were incubated at $37^{\circ} \mathrm{C}$ in $5 \% \mathrm{CO}_{2}$. All experiments were carried out using cells harvested at low $(<20)$ passages. To obtain KSHV for infection experiments, BCBL-1 cells were incubated with $0.6 \mathrm{mM}$ valproic acid for 6 days, and purified virus was concentrated from culture supernatants and infectious titers were determined as described previously [46]. For overexpression of CD147, HUVEC were transduced as previously described with a recombinant adenoviral vector (MOI 10) encoding CD147 (AdV-CD147), or a control vector $(\mathrm{AdV})$, for $48 \mathrm{~h}$ prior to subsequent analyses [11].

\section{Microarray}

Total RNA was isolated using Qiagen RNeasy kit (Qiagen), and $500 \mathrm{ng}$ of total RNA was used to synthesize dscDNA. Biotin-labeled RNA was generated using the TargetAmp-Nano Labeling Kit for Illumina Expression
BeadChip (Epicentre), according to the manufacturers' instructions, and hybridized to the HumanHT-12 v4 Expression BeadChip (Illumina), which contains more than 47,000 probes derived from the NCBI RefSeq Release 38 and other sources, at $58^{\circ} \mathrm{C}$ for $16 \mathrm{~h}$. The chip was washed, stained with streptavadin-Cy3, and scanned with the Illumina BeadStation 500 and BeadScan. Using the Illumina's GenomeStudio software, we normalized the signals using the "cubic spline algorithm" that assumes that the distribution of transcript abundance is similar in all samples, according to the method proposed by Workman et al. [47]. The background signal was removed using the "detection $p$-value algorithm" to remove targets with signal intensities equal or lower than that of irrelevant probes (with no known targets in the human genome but thermodynamically similar to the relevant probes). The microarray experiments were performed twice for each group and the average values were used for analysis. Common and unique sets of genes and enrichment analysis were performed using the MetaCore Software (Thompson Reuters) as previously reported [21]. The microarray original data have been submitted to Gene Expression Omnibus (GEO) database (Accession number: GSE69067).

\section{RNA interference}

CD147, ADAMTS1 or ADAMTS9 ON-TARGET plus SMART pool siRNA, or negative control siRNA (n-siRNA) (Dharmacon), were delivered using the DharmaFECT transfection reagent according to the manufacturer's instructions.

\section{Immunoblotting}

Total cell lysates $(20 \mu \mathrm{g})$ were resolved by $10 \%$ SDS-PAGE, transferred to nitrocellulose membranes, and immunoblotted with antibodies for CD147 (BD), ADAMTS1, ADAMTS9 (Cell Signaling) and $\beta$-Actin (Sigma) for loading controls. Immunoreactive bands were identified using an enhanced chemiluminescence reaction (Perkin-Elmer), visualized by autoradiography and quantitated using Image-J software.

\section{Immunofluorescence}

Cells were incubated in 1:1 methanol-acetone at $-20^{\circ} \mathrm{C}$ for fixation and permeabilization, then with a blocking reagent $(10 \%$ normal goat serum, 3\% bovine serum albumin, and $1 \%$ glycine) for an additional 30 minutes. Cells were then incubated for $1 \mathrm{~h}$ at $25^{\circ} \mathrm{C}$ with $1: 1000$ dilution of a rat anti-LANA monoclonal antibody (ABI) followed by 1:200 dilution of a goat anti-rat secondary antibody conjugated to Texas Red (Invitrogen). For identification of nuclei, cells were subsequently counterstained with $0.5 \mathrm{mg} / \mathrm{mL}$ 4',6-diamidino-2phenylindole (DAPI; Sigma) in $180 \mathrm{mM}$ Tris- $\mathrm{HCl}$ 
(pH 7.5). Slides were washed once in $180 \mathrm{mM}$ Tris- $\mathrm{HCl}$ for 15 minutes and prepared for visualization using a Leica TCPS SP5 AOBS confocal microscope.

\section{qRT-PCR}

Total RNA was isolated using the RNeasy Mini kit (QIAGEN), and cDNA was synthesized from equivalent total RNA using a SuperScript III First-Strand Synthesis SuperMix Kit (Invitrogen) according to the manufacturer's instructions. Primers used for amplification of target genes are displayed in Supplementary Table 1. Amplification was carried out using an iCycler IQ Real-Time PCR Detection System, and cycle threshold $(\mathrm{Ct})$ values were tabulated in duplicate for each gene of interest in each experiment. "No template" (water) controls were used to ensure minimal background contamination. Using mean $\mathrm{Ct}$ values tabulated for each gene, and paired $\mathrm{Ct}$ values for $\beta$-actin as a loading control, fold changes for experimental groups relative to assigned controls were calculated using automated iQ5 2.0 software (Bio-rad).

\section{ELISA}

Concentrations of IL-6 and VEGF in culture supernatants were determined using human IL-6 (eBioscience) and VEGF-A (Pierce Biotechnology) ELISA kits according to the manufacturers' instructions.

\section{Transwell invasion assays}

Matrigel Invasion Chambers (BD) were hydrated for $4 \mathrm{~h}$ at $37^{\circ} \mathrm{C}$ with culture media. Following hydration, media in the bottom of the well was replaced with fresh media, then $2 \times 10^{4}$ HUVEC, TIVE or TIVE-LT cells were plated in the top of the chamber. After $24 \mathrm{~h}$, cells were fixed with $4 \%$ formaldehyde for $15 \mathrm{~min}$ at room temperature and chambers rinsed in PBS prior to staining with $0.2 \%$ crystal violet for $10 \mathrm{~min}$. After washing the chambers, cells at the top of the membrane were removed and cells at the bottom of the membrane counted using a phase contrast microscope. Relative invasion was determined for cells in experimental groups as follows: relative invasion $=\#$ invading cells in experimental group / \# invading cells in control groups.

\section{Soft agar assays}

A base layer containing $0.5 \%$ agarose medium and $5 \%$ FCS was poured into six-well plates. Then, 10,000 cells were mixed with $0.4 \%$ agarose in Earl's minimal essential medium (EMEM) containing 5\% FCS to form a single-cell suspension. After being seeded, the plates were incubated for 2 weeks. Colonies were stained with $0.005 \%$ crystal violet and photographed under a phase-contrast microscope (Leica DFC320).

\section{KS-like nude mouse model}

Cells were counted and washed once in ice-cold PBS, and $5 \times 10^{5}$ cells in $50 \mu \mathrm{L}$ PBS plus $50 \mu \mathrm{L}$ growth factor-depleted Matrigel (BD Biosciences) were injected subcutaneously into the flanks of nude mice (Jackson Laboratory). The mice were observed and measured every $2 \sim 3 \mathrm{~d}$ for the presence of palpable tumors. At the end of experiment, the tumors were excised from the site of injection for subsequent analysis such as immunoblots and immunohistochemistry. All protocols were approved by the LSUHSC Animal Care and Use Committee in accordance with national guidelines.

\section{KS tumor tissues from $\mathrm{HIV}^{+}$patients and immunohistochemistry}

KS tissues from HIV-infected patients were provided by the LSUHSC HIV Outpatient (HOP) Clinic and Biospecimens Bank (LSUHSC IRB approved No. 8079). Formalin-fixed, paraffin-embedded tissues were microtome-sectioned to a thickness of $4 \mathrm{uM}$, placed on electromagnetically charged slides (Fisher Scientific), and stained with hematoxylin \& eosin (H \& E) for routine histologic analysis. Immunohistochemistry was performed using the Avidin-Biotin-Peroxidase complex system, according to the manufacturer's instructions (Vectastain Elite ABC Peroxidase Kit; Vector Laboratories). In our modified protocol, sections were deparaffinized in xylene and re-hydrated through a descending alcohol gradient. For non-enzymatic antigen retrieval, slides were heated in $0.01 \mathrm{M}$ sodium citrate buffer $(\mathrm{pH} 6.0)$ to $95^{\circ} \mathrm{C}$ under vacuum for $40 \mathrm{~min}$ and allowed to cool for $30 \mathrm{~min}$ at room temperature, then rinsed with PBS and incubated in $\mathrm{MeOH} / 3 \% \mathrm{H}_{2} \mathrm{O}_{2}$ for 20 min to quench endogenous peroxidase. Slides were then washed with PBS and blocked with $5 \%$ normal goat serum in $0.1 \%$ PBS/BSA for $2 \mathrm{~h}$ at room temperature, then incubated overnight with indicated antibody at 1:200-1:400 dilution in 0.1\% PBS/BSA. The following day, slides were incubated with appropriate secondary antibody at room temperature for $1 \mathrm{~h}$, followed by avidin-biotin peroxidase complexes for $1 \mathrm{~h}$ at room temperature. Finally, slides were developed using a diaminobenzidine substrate, counterstained with hematoxylin, dehydrated through an ascending alcohol gradient, cleared in xylene, and coverslipped with Permount. Images were collected using an Olympus BX61 microscope equipped with a high resolution DP72 camera and CellSense image capture software.

\section{Statistical analysis}

Significance for differences between experimental and control groups was determined using the two-tailed Student's $t$-test (Excel 8.0), and $p$ values $<0.05$ were considered significant. 


\section{ACKNOWLEDGMENTS AND FUNDINGS}

We thank Dr. Rolf Renne at University of Florida for his kind gifts of TIVE-LT and TIVE cells. This work was supported by grants from a DOD Career Development Award (CA140437), the SOM Research Enhancement Funding, as well as awards from the National Natural Science Foundation of China (81272191, 81472547 and 81400164 ) and the Foundation for Innovative Research Groups of the NNSF of China (81221001). De-identified human KS tumors were provided by the HIV Cancer Care Program Biorepository which is supported by grants from the National Institute of Health (UM1-CA181255 and R01-CA142362). Funding sources had no role in study design, data collection and analysis, decision to publish, or preparation of the manuscript.

\section{CONFLICTS OF INTEREST}

All the authors declare no conflicts of interest.

\section{REFERENCES}

1. Chang Y, Cesarman E, Pessin MS, Lee F, Culpepper J, Knowles DM, Moore PS. Identification of herpesvirus-like DNA sequences in AIDS-associated Kaposi's sarcoma. Science. 1994; 266:1865-1869.

2. Engels EA, Biggar RJ, Hall HI, Cross H, Crutchfield A, Finch JL, Grigg R, Hylton T, Pawlish KS, McNeel TS, Goedert JJ. Cancer risk in people infected with human immunodeficiency virus in the United States. Int J Cancer. 2008; 123:187-194.

3. Bonnet F, Lewden C, May T, Heripret L, Jougla E, Bevilacqua S, Costagliola D, Salmon D, Chene G, Morlat P. Malignancy-related causes of death in human immunodeficiency virus-infected patients in the era of highly active antiretroviral therapy. Cancer. 2004; 101:317324.

4. Jenkins FJ, Hoffman LJ, Liegey-Dougall A. Reactivation of and primary infection with human herpesvirus 8 among solid-organ transplant recipients. J Infect Dis. 2002; 185:1238-1243.

5. Luppi M, Barozzi P, Santagostino G, Trovato R, Schulz TF, Marasca R, Bottalico D, Bignardi L, Torelli G. Molecular evidence of organ-related transmission of Kaposi sarcomaassociated herpesvirus or human herpesvirus- 8 in transplant patients. Blood. 2000; 96:3279-3281.

6. Ariza-Heredia EJ, Razonable RR. Human herpes virus 8 in solid organ transplantation. Transplantation. 2011; 92: 837-844.

7. Biswas C, Zhang Y, DeCastro R, Guo H, Nakamura T, Kataoka H, Nabeshima K. The human tumor cell-derived collagenase stimulatory factor (renamed EMMPRIN) is a member of the immunoglobulin superfamily. Cancer Res. $1995 ; 55: 434-439$.

8. Yan L, Zucker S, Toole BP. Roles of the multifunctional glycoprotein, emmprin (basigin; CD147), in tumour progression. Thromb Haemost. 2005; 93:199-204.

9. Grass GD, Dai L, Qin Z, Parsons C, Toole BP. CD147: regulator of hyaluronan signaling in invasiveness and chemoresistance. Adv Cancer Res. 2014; 123:351-373.

10. Qin Z, Dai L, Slomiany MG, Toole BP, Parsons C. Direct activation of emmprin and associated pathogenesis by an oncogenic herpesvirus. Cancer Res. 2010; 70:3884-3889.

11. Dai L, Bratoeva M, Toole BP, Qin Z, Parsons C. KSHV activation of VEGF secretion and invasion for endothelial cells is mediated through viral up-regulation of emmprininduced signal transduction. Int J Cancer. 2012; 131: 834-843.

12. Qin Z, Dai L, Bratoeva M, Slomiany MG, Toole BP, Parsons C. Cooperative roles for emmprin and LYVE-1 in the regulation of chemoresistance for primary effusion lymphoma. Leukemia. 2011; 25:1598-1609.

13. An FQ, Folarin HM, Compitello N, Roth J, Gerson SL, McCrae KR, Fakhari FD, Dittmer DP, Renne R. Long-terminfected telomerase-immortalized endothelial cells: a model for Kaposi's sarcoma-associated herpesvirus latency in vitro and in vivo. J Virol. 2006; 80:4833-4846.

14. McAllister SC, Hansen SG, Ruhl RA, Raggo CM, DeFilippis VR, Greenspan D, Fruh K, Moses AV. Kaposi sarcoma-associated herpesvirus (KSHV) induces heme oxygenase-1 expression and activity in KSHV-infected endothelial cells. Blood. 2004; 103:3465-3473.

15. Alcendor DJ, Knobel S, Desai P, Zhu WQ, Hayward GS. KSHV regulation of fibulin-2 in Kaposi's sarcoma: implications for tumorigenesis. Am J Pathol. 2011; 179:1443-1454.

16. Cross NA, Chandrasekharan S, Jokonya N, Fowles A, Hamdy FC, Buttle DJ, Eaton CL. The expression and regulation of ADAMTS-1, -4, -5, -9, and -15, and TIMP-3 by TGFbeta1 in prostate cells: relevance to the accumulation of versican. Prostate. 2005; 63:269-275.

17. Ricciardelli C, Frewin KM, Tan Ide A, Williams ED, Opeskin K, Pritchard MA, Ingman WV, Russell DL. The ADAMTS1 protease gene is required for mammary tumor growth and metastasis. Am J Pathol. 2011; 179:3075-3085.

18. Okada Y. Tumor cell-matrix interaction: pericellular matrix degradation and metastasis. Verh Dtsch Ges Pathol. 2000; $84: 33-42$.

19. Demircan K, Gunduz E, Gunduz M, Beder LB, Hirohata S, Nagatsuka H, Cengiz B, Cilek MZ, Yamanaka N, Shimizu K, Ninomiya Y. Increased mRNA expression of ADAMTS metalloproteinases in metastatic foci of head and neck cancer. Head Neck. 2009; 31:793-801. 
20. Tan Ide A, Ricciardelli C, Russell DL. The metalloproteinase ADAMTS1: a comprehensive review of its role in tumorigenic and metastatic pathways. Int J Cancer. 2013; 133:2263-2276.

21. Kim SH, Sierra RA, McGee DJ, Zabaleta J. Transcriptional profiling of gastric epithelial cells infected with wild type or arginase-deficient Helicobacter pylori. BMC Microbiol. $2012 ; 12: 175$

22. Cavallin LE, Goldschmidt-Clermont P, Mesri EA. Molecular and cellular mechanisms of KSHV oncogenesis of Kaposi's sarcoma associated with HIV/AIDS. PLoS Pathog. 2014; 10:e1004154.

23. Mesri EA, Feitelson MA, Munger K. Human viral oncogenesis: a cancer hallmarks analysis. Cell Host Microbe. 2014; 15:266-282.

24. Mesri EA, Cesarman E, Boshoff C. Kaposi's sarcoma and its associated herpesvirus. Nat Rev Cancer. 2010; 10: 707-719.

25. Riva G, Barozzi P, Torelli G, Luppi M. Immunological and inflammatory features of Kaposi's sarcoma and other Kaposi's sarcoma-associated herpesvirus/human herpesvirus 8-associated neoplasias. AIDS Rev. 2010; $12: 40-51$.

26. Ensoli B, Sturzl M. Kaposi's sarcoma: a result of the interplay among inflammatory cytokines, angiogenic factors and viral agents. Cytokine Growth Factor Rev. 1998; 9:63-83.

27. Sivakumar R, Sharma-Walia N, Raghu H, Veettil MV, Sadagopan S, Bottero V, Varga L, Levine R, Chandran B. Kaposi's sarcoma-associated herpesvirus induces sustained levels of vascular endothelial growth factors A and C early during in vitro infection of human microvascular dermal endothelial cells: biological implications. J Virol. 2008; 82:1759-1776.

28. Qian LW, Xie J, Ye F, Gao SJ. Kaposi's sarcoma-associated herpesvirus infection promotes invasion of primary human umbilical vein endothelial cells by inducing matrix metalloproteinases. J Virol. 2007; 81:7001-7010.

29. Ballestas ME, Chatis PA, Kaye KM. Efficient persistence of extrachromosomal KSHV DNA mediated by latencyassociated nuclear antigen. Science. 1999; 284:641-644.

30. Dai L, Chen Y, Toole B, Parsons C, Qin Z. Induction of hyaluronan production by oncogenic KSHV and the contribution to viral pathogenesis in AIDS patients. Cancer Lett. 2015; 362:158-166.

31. Cheng F, Pekkonen P, Laurinavicius S, Sugiyama N, Henderson S, Gunther T, Rantanen V, Kaivanto E, Aavikko M, Sarek G, Hautaniemi S, Biberfeld P, Aaltonen L, et al. KSHV-initiated notch activation leads to membrane-type-1 matrix metalloproteinase-dependent lymphatic endothelial-to-mesenchymal transition. Cell Host Microbe. 2011; 10:577-590.
32. Gasperini P, Espigol-Frigole G, McCormick PJ, Salvucci O, Maric D, Uldrick TS, Polizzotto MN, Yarchoan R, Tosato G. Kaposi sarcoma herpesvirus promotes endothelial-to-mesenchymal transition through Notchdependent signaling. Cancer Res. 2012; 72:1157-1169.

33. Wu J, Ru NY, Zhang Y, Li Y, Wei D, Ren Z, Huang XF, Chen ZN, Bian H. HAb18G/CD147 promotes epithelialmesenchymal transition through TGF-beta signaling and is transcriptionally regulated by Slug. Oncogene. 2011; 30:4410-4427.

34. Grass GD, Bratoeva M, Toole BP. Regulation of invadopodia formation and activity by CD147. J Cell Sci. 2012; 125:777-788.

35. Grass GD, Tolliver LB, Bratoeva M, Toole BP. CD147, CD44, and the epidermal growth factor receptor (EGFR) signaling pathway cooperate to regulate breast epithelial cell invasiveness. J Biol Chem. 2013; 288:26089-26104.

36. Wang J, Fu L, Gu F, Ma Y. Notch1 is involved in migration and invasion of human breast cancer cells. Oncol Rep. $2011 ; 26: 1295-1303$.

37. Quemener C, Gabison EE, Naimi B, Lescaille G, Bougatef F, Podgorniak MP, Labarchede G, Lebbe C, Calvo F, Menashi S, Mourah S. Extracellular matrix metalloproteinase inducer up-regulates the urokinasetype plasminogen activator system promoting tumor cell invasion. Cancer Res. 2007; 67:9-15.

38. Kuno K, Matsushima K. ADAMTS-1 protein anchors at the extracellular matrix through the thrombospondin type I motifs and its spacing region. J Biol Chem. 1998; 273:13912-13917.

39. Hatipoglu OF, Hirohata S, Cilek MZ, Ogawa H, Miyoshi T, Obika M, Demircan K, Shinohata R, Kusachi S, Ninomiya $\mathrm{Y}$. ADAMTS1 is a unique hypoxic early response gene expressed by endothelial cells. J Biol Chem. 2009; 284:16325-16333.

40. Ke X, Fei F, Chen Y, Xu L, Zhang Z, Huang Q, Zhang H, Yang H, Chen Z, Xing J. Hypoxia up-regulates CD147 through a combined effect of HIF-1alpha and Sp1 to promote glycolysis and tumor progression in epithelial solid tumors. Carcinogenesis. 2012; 33:1598-1607.

41. Cai Q, Lan K, Verma SC, Si H, Lin D, Robertson ES. Kaposi's sarcoma-associated herpesvirus latent protein LANA interacts with HIF-1 alpha to up-regulate RTA expression during hypoxia: Latency control under low oxygen conditions. J Virol. 2006; 80:7965-7975.

42. Ma Q, Cavallin LE, Yan B, Zhu S, Duran EM, Wang H, Hale LP, Dong C, Cesarman E, Mesri EA, GoldschmidtClermont PJ. Antitumorigenesis of antioxidants in a transgenic Rac1 model of Kaposi's sarcoma. Proc Natl Acad Sci U S A. 2009; 106:8683-8688.

43. Shin YC, Joo CH, Gack MU, Lee HR, Jung JU. Kaposi's sarcoma-associated herpesvirus viral IFN regulatory factor 3 
stabilizes hypoxia-inducible factor-1 alpha to induce vascular endothelial growth factor expression. Cancer Res. 2008; 68:1751-1759.

44. Sodhi A, Montaner S, Patel V, Zohar M, Bais C, Mesri EA, Gutkind JS. The Kaposi's sarcoma-associated herpes virus $G$ protein-coupled receptor up-regulates vascular endothelial growth factor expression and secretion through mitogen-activated protein kinase and p38 pathways acting on hypoxia-inducible factor 1alpha. Cancer Res. 2000; 60:4873-4880.

45. Dai L, Trillo-Tinoco J, Bai L, Kang B, Xu Z, Wen X, Del Valle L, Qin Z. Systematic analysis of a xenograft mice model for $\mathrm{KSHV}+$ primary effusion lymphoma (PEL). PLoS One. 2014; 9:e90349.
46. Qin Z, Dai L, Defee M, Findlay VJ, Watson DK, Toole BP, Cameron J, Peruzzi F, Kirkwood K, Parsons C. Kaposi's sarcoma-associated herpesvirus suppression of DUSP1 facilitates cellular pathogenesis following de novo infection. J Virol. 2013; 87:621-635.

47. Workman C, Jensen LJ, Jarmer H, Berka R, Gautier L, Nielser HB, Saxild HH, Nielsen C, Brunak S, Knudsen S. A new non-linear normalization method for reducing variability in DNA microarray experiments. Genome Biol. 2002; 3:research0048. 